\title{
EDITORIAL
}

Check for updates

\section{From exploration to enterprise}

\author{
As a field moves from fundamental science to industrial applications, standards for \\ reproducibility also evolve. We commit to provide a platform for this ongoing conversation.
}

This month, we publish an Expert Recommendation on making clean electrical contacts on 2D semiconductors. Although 2D materials has been a very active field of research over the past 15 years, making electrical contact to them in a consistent way is just one of many challenges facing the field today. From an industry point of view, this lack of reproducibility is a deal breaker. However, from a fundamental physics perspective, many interesting new phenomena have been observed in a few lucky devices, which have provided insight into the workings of these materials. As the field of 2D materials develops, and researchers have an eye towards industrial applications, the bar for reproducible standards must also evolve.

With the exfoliation of graphene and the advent of monolayer materials, a whole field of research opened up to the condensed-matter community, ripe for exploratory studies. 2D materials are notoriously sensitive to growth conditions, processing techniques and even sustained measurement over multiple thermal cycles. This sensitivity means that almost every device is different, enabling researchers to measure a variety of systems quickly. However, once an interesting device is found, it is not trivial to replicate it to corroborate results. Because a lot of the initial research has taken place in small lab settings, often the equipment used in the experiments is bespoke and unique to each lab. This lack of standardized equipment makes it challenging to replicate results between groups.

The situation becomes even more complex with multilayer systems. Combining monolayers of 2D materials, often with relative twists between then, can lead to interesting emergent phenomena. However, a myriad of factors can affect the physical properties of a $2 \mathrm{D}$ heterostructure including twist angle, strain, defects, external crystal alignment and pressure, all of which are very hard to replicate identically. Not only is it difficult to know how many samples out of a batch are suitably twisted to display the desired properties, but even measuring the precise twist angle remains a challenge. Samples are also inhomogenous and often different parts of the same sample will show variable results. It is often difficult to compare results from different groups, as there is no standard technique for characterizing the interface between two layers.

Although reproducibility is on the mind of the scientific community doing fundamental research, the level of scalability and reproducibility needed in industry is much higher. Graphene is furthest along the path to commercialization, yet even so, there remains a lack of clarity around reproducibility. The hundreds of commercial suppliers for graphene use a variety of measurands on technical data sheets, making it impossible to compare across providers. In 2021, the first international measurement standard for graphene was published. In a Comment we published last year to coincide with the standard, Charles Clifford and colleagues lay out the importance of standardizing measurements for graphene and other 2D materials for use in industry.

However, in the case of newer materials that are being studied in small labs, insisting on strictly reproducible results over a statistically significant number of devices or samples would hamper many results from being published and shared. In 2020, we hosted a panel discussion on this topic, featuring Joshua Lui (UC Riverside), Cecilia Mattevi (Imperial College London) and Matthew Yankowitz (University of Washington), moderated by Giulia Pacchioni (Nature Reviews Materials). During the discussion, researchers and editors both agreed that this would greatly slow down the growth of the field. Rather, what is needed is greater transparency over every aspect of the experimental procedure - from the type of equipment used, to details on exactly how a particular device was handled.

As the field evolves from the exploratory phase to enterprise, the standards of reproducibility needed by the community will change. As a Reviews journal, our role is to support conversations around issues like reproducibility and rigour. We want to provide a platform for analysis and insight into discussions that may otherwise be relegated to the methods or supplementary information of research papers. 